\title{
Industry updates from the field of stem cell research and regenerative medicine in November 2019
}

\author{
Dusko llic*,1(i) \& Amyra Binti Mohd Amran De Costa ${ }^{2}$ \\ ${ }^{1}$ Stem Cell Laboratories, Guy's Assisted Conception Unit, Department of Women \& Children's Health, Faculty of Life Sciences \& \\ Medicine, King's College London, London, UK \\ ${ }^{2}$ MSc Stem Cell \& Regenerative Therapies: from Bench to Market, Faculty of Life Sciences \& Medicine, King's College London, \\ London, UK \\ *Author for correspondence: dusko.ilic@kcl.ac.uk
}

Latest developments in the field of stem cell research and regenerative medicine compiled from publicly available information and press releases from nonacademic institutions in November 2019.

First draft submitted: 29 December 2019; Accepted for publication: 30 January 2020; Published online: 14 February 2020

Keywords: industry • regenerative medicine $\bullet$ stem cells

\section{Business development}

Collaborations, partnerships \& alliances

Collaboration agreement: Allogene \& Notch

Allogene Therapeutics (CA, USA; www.allogene.com), a clinical-stage biotechnology company pioneering the development of allogeneic CAR T (AlloCAR $\mathrm{T}^{\mathrm{TM}}$ ) therapies for cancer, and Notch Therapeutics (ON, Canada; www.notchtx.com), an immune cell therapy company creating universally compatible, allogeneic T-cell therapies for the treatment of diseases of high unmet need, have announced an exclusive collaboration and license agreement to research and develop induced pluripotent stem cell (iPSC) AlloCAR therapy products for initial application in non-Hodgkin lymphoma, leukemia and multiple myeloma [1]. Under the partnership, Allogene and Notch will create allogeneic cell therapy candidates from T cells or natural killer (NK) cells using Notch's Engineered Thymic Niche (ETN) platform. ETN platform drives the expansion and differentiation of iPSC in scalable, fully defined, feeder-free and serum-free cultures into $\mathrm{T}$ cells that can be genetically tailored for any T-cell-based immunotherapeutic application.

Under the terms of the agreement, Notch will be responsible for preclinical research of next-generation iPSC AlloCAR T cells. Allogene will clinically develop the product candidates and holds exclusive worldwide rights to commercialize resulting products. Allogene will provide to Notch an upfront payment of USD 10 million. Notch will be eligible to receive up to USD 7.25 million upon achieving certain agreed research milestones, up to USD 4.0 million per exclusive target upon achieving certain preclinical development milestones, and up to USD 283 million per exclusive target and cell type upon achieving certain clinical, regulatory and commercial milestones as well as tiered royalties on net sales in the mid-to-high single digits. In addition to this collaboration and license agreement, Allogene has acquired a $25 \%$ equity position in Notch and will assume a seat on Notch's Board of Directors.

\section{Collaboration agreement: Avita \& Gates Center}

AVITA Medical (Australia; www.avitamedical.com), a regenerative medicine company with a technology platform positioned to address unmet medical needs in therapeutic skin restoration, and scientists at the Gates Center for Regenerative Medicine at the University of Colorado School of Medicine (CO, USA; https://medschool.cuansc hutz.edu/gates-center-for-regenerative-medicine/home/1) have announced a preclinical research collaboration to establish proof-of-concept and explore further development of a spray-on treatment of genetically modified cells 
for patients with epidermolysis bullosa (EB), with potential applicability to other genetic skin disorders [2]. The partnership will pair AVITA Medical's patented and proprietary Spray-On Skin ${ }^{T M}$ Cells technology and expertise with the Gates Center's innovative, patent-pending combined reprogramming and gene-editing technology to allow cells to function properly. Under the terms of the Sponsored Research Agreement, AVITA Medical retains the option to exclusively license technologies emerging from the partnership for further development and commercialization. The Gates Center team is further supported by the EB Research Partnership in New York, the Los Angeles-based EB Medical Research Foundation, the London-based Cure EB Charity and government grants in a collaborative effort to rapidly develop and translate this technology to the clinic for meaningful impact on patient lives.

\section{Collaboration agreement: Bluebird \& Forty Seven}

Bluebird Bio (MA, USA; www.bluebirdbio.com) and Forty Seven (CA, USA; www.fortyseveninc.com) have entered into a research collaboration to pursue clinical proof-of-concept for Forty Seven's novel antibody-based conditioning regimen, FSI-174 (anti-cKIT antibody) plus magrolimab (anti-CD47 antibody), with Bluebird's ex vivo lentiviral vector hematopoietic stem cell (LVV HSC) gene therapy platform [3]. This collaboration will focus on a conditioning approach aimed to deliver reduced toxicity and will initially target diseases that have the potential to be corrected with transplantation of autologous gene-modified blood-forming stem cells. If successful, the new conditioning regimen could allow for more patients to undergo gene therapy.

Autologous hematopoietic stem cell transplantation (HSCT) and most ex vivo LVV HSC gene therapies require that a patient's own stem cells first be depleted from the bone marrow to facilitate the engraftment of the new (or gene-modified) HSCs through a process called conditioning. Conditioning is performed using chemotherapy or radiation, which can place patients at risk for infection and require hospitalization until bone marrow cells have recovered. In addition, conventional conditioning can place patients at risk for secondary malignancy and infertility. As a result, the overall toxicity profile of current conditioning regimens limits the types of patients who are eligible for gene therapy. It is hoped that novel antibody based conditioning regimens could avoid these toxicities.

\section{Collaboration agreement: Editas \& Celgene}

Editas Medicine (MA, USA; www.editasmedicine.com), a genome editing company, has amended collaboration with Celgene Corporation (NJ, USA; www.celgene.com) under which the parties may research, develop and commercialize autologous and allogeneic alpha-beta T-cell medicines for the treatment of cancer and autoimmune diseases [4]. Under the terms of the amended agreement, Editas Medicine will receive a payment of USD 70 million.

\section{Collaboration agreement: Novoheart \& AstraZeneca}

Novoheart (BC, Canada; www.novoheart.com) has announced a collaboration with AstraZeneca (UK; www.astraz eneca.com), in an effort to develop the world's first human-specific in vitro, functional model of heart failure with preserved ejection fraction ( $\mathrm{HFpEF}$ ), a common condition especially among the elderly and in women, with the reported prevalence approaching $10 \%$ in women over the age of 80 years [5]. The initial phase of the project aims to establish a new in vitro model, leveraging Novoheart's proprietary $3 \mathrm{D}$ human ventricular cardiac organoid chamber (hvCOC) technology that reproduces key phenotypic characteristics of HFpEF. Also known as 'human heart-in-ajar', the hvCOC is the only human engineered heart tissue available on the market to date that enables clinically informative assessment of human cardiac pump performance including ejection fraction and developed pressure. Unlike animal models, engineered hvCOCs can be fabricated with specific cellular and matrix compositions, and patient-specific iPSCs, that allow control over their physical and mechanical properties to mimic those observed in HFpEF patient hearts. Together with Novoheart's proprietary hardware and software, this aims to provide a unique assay for understanding the mechanisms of HFpEF, identification of new therapeutic targets and assessment of novel therapeutics for treating HFpEF patients. Novoheart will exclusively own the intellectual property rights to the newly developed HFpEF hvCOC model.

\section{Collaboration agreement: Tmunity \& Oncora}

Tmunity Therapeutics (PA, USA; www.tmunity.com), a private clinical-stage biotherapeutics company focused on T-cell immunotherapy, has announced it has entered into a strategic collaboration with Oncora Medical (PA, USA; www.oncoramedical.com) to access Oncora's proprietary real-world data platform to support the design of future clinical programs with cell therapy [6]. Oncora brings to this collaboration a suite of intuitive analytics software that collects and deploys real-world clinical data that can be used to drive better decision making and treatment options 
and ultimately improve patient outcomes. Tmunity brings expertise in clinical oncology and clinical trial design, regulatory strategy and cell therapy. Together, this collaboration has the potential to innovate the way clinical trials for cell therapy are executed to bring treatments to patients more efficiently. Tmunity will leverage Oncora's data and analytics capabilities to refine, customize and validate a real-world evidence strategy. Tmunity hopes to validate the strategy in a clinical program selected from its highly innovative pipeline.

\section{License agreement: Takeda \& MD Anderson}

The University of Texas MD Anderson Cancer Center (TX, USA; www.mdanderson.org) and Takeda Pharmaceutical (Japan; www.takeda.com) have announced an exclusive license agreement and research agreement to develop cord blood derived chimeric antigen receptor-directed natural killer (CAR NK)-cell therapies, 'armored' with IL-15, for the treatment of B-cell malignancies and other cancers [7]. Under the agreement, Takeda will receive access to MD Anderson's CAR NK platform and the exclusive rights to develop and commercialize up to four programs, including a CD19-targeted CAR NK-cell therapy and a B-cell maturation antigen (BCMA)-targeted CAR NK-cell therapy. Takeda and MD Anderson will also conduct a research collaboration to further develop these CAR NK programs.

\section{Achievements}

\section{Emulate}

Emulate (MA, USA: www.emulatebio.com), a privately held company that develops a wide range of Organ-Chips and disease models, has announced the publication of research demonstrating that its Liver-Chip accurately modeled and predicted human toxicity in a range of eight previously studied drugs [8,9]. Results from the Liver-Chip study showed the ability to model the mechanism of action for hepatotoxicity, measure relevant clinical biomarkers and predict species differences between human, dog and rat liver drug toxicity for the drugs tested. The collaborative research supports efforts to better predict drug safety and human response in the drug development process, as well as, initiatives for $3 \mathrm{Rs}$ programs for the reduction, refinement and replacement of animal testing. The liver is among the organ systems most commonly affected by adverse drug reactions, and drug-induced liver injury is one of most frequent reasons for the withdrawal of drugs from the market.

\section{Launching new products, services...}

\section{Chiesi}

The first patient, funded by the UK's NHS, has received treatment with Chiesi's (Italy; www.chiesi.com) stemcell-based therapy Holoclar for limbal stem cell deficiency caused by chemical eye burns [10]. The patient received treatment at Newcastle upon Tyne Hospitals NHS Foundation Trust. Limbal stem cell deficiency is a rare eye disorder, which could result in chronic pain, photophobia, burning, inflammation, stromal scarring, corneal neovascularization and even blindness. Chiesi's Holoclar is a tissue-engineered product, made of cells obtained from the patient's healthy limbus during a biopsy. The extracted cells are sent to the manufacturing facility in Italy to be prepared and grown in a culture to generate a layer of healthy tissue. After a minimum of 50 days, the healthy tissue layer is transported back to the treating hospital for implantation into the damaged eye to repair the damaged corneal surface.

\section{HemaCare}

HemaCare Corporation (CA, USA; www.hemacare.com) has expanded its portfolio of GMP-compliant cellular material with the launch of GMPrime bone marrow aspirate [11]. GMPrime bone marrow aspirate is collected within HemaCare's US FDA-registered collection center from IRB-consented donors following rigorous and standardized processes to ensure the highest achievable purity, viability and quality. HemaCare, with over 40 years of donor management experience, possesses one of the industry's largest reliable pedigreed donor networks. By expanding its GMP portfolio, researchers can now choose GMP-compliant bone marrow aspirate as they transition from preclinical to clinical development, thus ensuring consistent, validated and fully documented quality.

\section{Kiadis}

Kiadis Pharma (the Netherlands; www.kiadis.com) is implementing a restructuring program to refocus the organization on its NK-cell therapy platform, which will result in a reduction of approximately half of its workforce, a reduction in external clinical trial costs associated with the Phase III study and a reduced company cash burn [12]. 
The company will discontinue development of ATIR101 and stop its ongoing Phase III trial. The company ended the third quarter of 2019 with approximately USD 52 (EUR 47) million of cash.

Kiadis' NK-cell program consists of off-the-shelf and haplo donor cell therapy products for the treatment of liquid and solid tumors. Kiadis' proprietary off-the-shelf NK-cell platform is based on NK cells from unique universal donors, expanded and activated ex vivo using our PM21 particle technology. The Kiadis off-the-shelf platform has the potential to make NK-cell therapy products rapidly and economically available for a broad patient population across a potentially wide range of indications.

The company's pipeline includes:

- K-NK002: A Phase I/II study will begin in 2020 evaluating K-NK002 as an adjunctive treatment to the current standard-of-care haploidentical hematopoietic stem cell transplantation with post-transplant cyclophosphamide (PTC-y). Relapse remains an issue with the PTC-y protocol. The Phase I/II study was designed based on promising clinical proof-of-concept data in 25 patients that demonstrated a reduction of long-term relapse rates from $45 \%$ in a matched contemporaneous control of patients treated with PTC-y, to $8 \%$ of patients treated with PTC-y and K-NK002. The 63-patient Phase I/II study will be conducted in collaboration with the Bone Marrow Transplant Clinical Trial Network (BMT-CTN), which consists of the premier transplant clinics in USA;

- K-NK003: A Phase I/IIA study will begin in 2020 evaluating K-NK003 as a treatment for patients with relapse and refractory acute myeloid leukemia. The trial is designed based on clinical proof-of-concept data that showed a $69 \%$ complete response rate;

- Pre-clinical programs: Kiadis has multiple preclinical programs evaluating its K-NK-cell therapies for the treatment of solid tumors.

\section{Takara Bio}

Takara Bio (Japan; www.takarabio.com) has launched the world's first commercially available small intestinal epithelial cells derived from human iPSC [13]. The new Cellartis ${ }^{\circledR}$ Intestinal Epithelial Cells (from ChiPSC18) Kit was jointly developed when Takara Bio received a technology transfer to the Osaka University. These hiPSC-derived cells express intestinal markers at levels comparable to that of adult small intestinal tissue. They also express drug metabolizing enzyme CYP3A4 and drug transporter PEPT1 at more physiologically relevant levels compared with Caco-2, the current gold standard of intestinal models. Additionally, the monolayer of intestinal epithelial cells forms a functional barrier that can be used for predicting intestinal permeability. Altogether, these cells provide an improved model for the study of absorption and metabolism of orally administered drugs. Previously made available in Japan, the cells are now available in USA through TakaraBio USA, formerly known as Clontech Laboratories.

\section{Clinical trials \\ Embryonic stem cells \\ Lineage Cell Therapeutics}

Lineage Cell Therapeutics (CA, USA; www.lineagecell.com) has provided an update on the Company's oligodendrocyte progenitor cell 1 (OPC1) therapy currently being tested in a Phase I/IIa clinical trial, the SCiStar Study (www.clinicaltrials.gov; ID: NCT02302157), for the treatment of acute spinal cord injury (SCI) [14].

- Overall safety profile of OPC1 for Year 2 follow-ups available to date (21 patients):

- Magnetic resonance imaging (MRI) scans at 24 months post-injection of OPC1 have shown no evidence of adverse changes in any of the 21 SCiStar study patients treated with OPC1 who have had their Year 2 follow-up visits;

- To date, there have been no unexpected serious adverse events (SAEs) related to the OPC1 cells in these patients, with no concerning safety issues noted;

- No SCiStar patient had a decline in their motor function from their Year 1 to Year 2 visits;

- No adverse findings were observed on follow-up MRI scans.

- Motor level improvements:

- For Cohort 1, even at a low dose of OPC1, patients continue to be stable 2-4 years out post treatment;

- Five of six Cohort 2 patients achieved at least two motor levels of improvement over baseline on at least one side as of their 24-month follow-up visit; 
- In addition, one Cohort 2 patient achieved three motor levels of improvement on one side over baseline as of the patient's 24-month follow-up visit; improvement has been maintained through the patient's 36-month follow-up visit;

- Improvements in upper extremity motor function are being measured using the International Standards for Neurological Classification of Spinal Cord Injury (ISNCSCI) scale, widely used to quantify functional status of patients with spinal cord injuries. Improvements in upper extremity motor function are the most desirable functional improvement target in the quadriplegic population, since even relatively modest changes can potentially have a significant impact on functional independence, quality of life and cost of care.

- Upper Extremity Motor Score:

- Improvements in Upper Extremity Motor Scores (UEMS) for Cohort 2 patients were observed at 24 months, with three patients who have recovered back to a UEMS in the mid-to-high 40's. The maximum total UEMS is 50 . The UEMS is a linear scale used to quantify motor function at each of five upper extremity muscle groups driving arm and hand function; these scores are also used to determine 'motor levels', which define the level within the cord above in which a patient has normal function.

- OPC1 Clinical Program Update:

- OPC1 manufacturing has now completely been transferred to the Company's cGMP manufacturing facility in Israel;

- Continued improvements to the manufacturing process are planned during 2020 and include enhancements to the production process to ensure robust, controlled and commercially viable scale, purity and reproducibility of OPC1;

- A meeting with the FDA is planned around the middle of 2020 to discuss the Company's proposed manufacturing improvements and the further development of OPC1 to best set the program up for success moving forward;

- Concurrently, Lineage will work to expand its partnerships with spinal cord injury advocacy and support organizations to support their mission to accelerate stem cell treatments to patients with unmet medical needs and fast-track the development of the most promising stem cell technologies.

\section{Hematopoietic stem cells}

CRISPR Therapeutics \& Vertex

CRISPR Therapeutics (Switzerland; www.crisprtx.com) and Vertex Pharmaceuticals Incorporated (MA, USA; www.vrtx.com) have announced positive, interim data from the first two patients with severe hemoglobinopathies treated with the investigational CRISPR/Cas9 gene-editing therapy CTX001 in ongoing Phase I/II clinical trials [15]. Patients who enroll in these studies have hematopoietic stem and progenitor cells collected from peripheral blood. The patient's cells are edited using the CRISPR/Cas9 technology. The edited cells, CTX001, are then infused back into the patient as part of a stem cell transplant, a process which involves, among other things, a patient being treated with myeloablative busulfan conditioning. Patients undergoing stem cell transplants may also encounter side effects, ranging from mild to severe, that are unrelated to the administration of CTX001. Patients are initially monitored to determine when the edited cells begin to produce mature blood cells, a process known as engraftment. After engraftment, patients are continued to be monitored to track the impact of CTX001 on multiple measures of disease.

One patient with transfusion-dependent beta thalassemia (TDT) received CTX001 in the first quarter of 2019 and data for this patient reflect 9 months of safety and efficacy follow-up. One patient with severe sickle cell disease (SCD) received CTX001 in mid-2019 and data for this patient reflect 4 months of safety and efficacy follow-up. These studies are ongoing, and patients will be followed for approximately 2 years following infusion. Several additional patients have been enrolled and have had drug product manufactured across the two studies.

The patient with TDT has the $\beta^{\circ} /$ IVS-I-110 genotype and required 16.5 transfusions per year (annualized rate during the 2 years prior to consenting for the study) before enrolling in the clinical study. The patient achieved neutrophil engraftment 33 days after CTX001 infusion and platelet engraftment 37 days after infusion. Two serious adverse events occurred, neither of which the principal investigator considered related to CTX001: pneumonia in the presence of neutropenia and veno-occlusive liver disease attributed to busulfan conditioning; both subsequently resolved. At 9 months after CTX001 infusion, the patient was transfusion independent and had total hemoglobin levels of $11.9 \mathrm{~g} / \mathrm{dl}, 10.1 \mathrm{~g} / \mathrm{dl}$ fetal hemoglobin and $99.8 \%$ F-cells (erythrocytes expressing fetal hemoglobin). 
The ongoing Phase I/II open-label trial, CLIMB-Thal-111, is designed to assess the safety and efficacy of a single dose of CTX001 in patients of 18-35 years of age with TDT. The study will enroll up to 45 patients and follow patients for approximately 2 years after infusion. Each patient will be asked to participate in a long-term follow-up study. Enrollment is ongoing at six clinical trial sites in USA, Canada and Europe.

The patient with SCD experienced seven vaso-occlusive crises per year (annualized rate during the 2 years prior to consenting for the study) before enrolling in the clinical study. The patient achieved neutrophil and platelet engraftment 30 days after CTX001 infusion. Three serious adverse events occurred, none of which the PI considered related to CTX001: sepsis in the presence of neutropenia, cholelithiasis and abdominal pain, all of which resolved. At 4 months after CTX001 infusion, the patient was free of vaso-occlusive crises and had total hemoglobin levels of $11.3 \mathrm{~g} / \mathrm{dl}$, 46.6\% fetal hemoglobin and 94.7\% F-cells (erythrocytes expressing fetal hemoglobin).

The ongoing Phase I/II open-label trial, CLIMB-SCD-121, is designed to assess the safety and efficacy of a single dose of CTX001 in patients of 18-35 years of age with severe SCD. The study will enroll up to 45 patients and follow patients for approximately 2 years after infusion. Each patient will be asked to participate in a long-term follow-up study. Enrollment is ongoing at 12 clinical trial sites in USA, Canada and Europe.

\section{T cells}

Cartesian

Cartesian Therapeutics (CA, USA; www.cartesiantherapeutics.com), a fully integrated, clinical-stage biopharmaceutical company developing cell and gene therapies for cancer and autoimmune diseases, has announced that it has initiated a Phase I/II clinical trial (https:/clinicaltrials.gov/; ID: NCT04146051) of its lead CAR-T candidate, Descartes-08, in patients with generalized myasthenia gravis [16]. To the company's knowledge, the program is the first CAR-T investigational candidate to enter clinical development for an autoimmune disease. Patients with severe generalized myasthenia gravis have limited treatment options and are often dependent on nonselective, chronic immunosuppressive therapies that have long-term toxicities. Cartesian's CAR-T technology selectively targets cells expressing BCMA, a protein expressed by all antibody-producing plasma cells. Such selective targeting would be a first in generalized myasthenia gravis and could help patients discontinue use of chronic immunosuppressive therapies.

\section{Regulations, approvals, acquisitions... Green light Hope Biosciences \& Altoida}

Hope Biosciences (TX, USA; www.hope.bio), a clinical stage biotechnology company focused on developing cellbased therapeutics, has announced that US FDA has approved a Phase I/II clinical trial evaluating safety and efficacy of Hope Biosciences' autologous, adipose-derived mesenchymal stem cells (HB-adMSCs) for Alzheimer's disease, in partnership with Altoida (TX, USA; https://altoida.com), a predictive digital biomarker company [17]. Altoida has created an application that utilizes augmented reality, machine learning and active Digital Biomarkers to classify patient's risk for mild cognitive impairment due to Alzheimer's disease. Altoida's novel technology, the Altoida Neuro Motor Index (NMI), is a medical device validated by 12 peer-reviewed publications and in clinical studies with more than 4000 patients, this FDA \& CE cleared Medical Device detects patterns of cognitive decline with $94 \%$ diagnostic accuracy between 6 and 10 years prior to the onset of symptoms. The gamified collection of functional and cognitive data by utilizing tablet or smartphone sensors takes 10 min only and is the world's first cognitive measurement of micro errors with active digital biomarkers. Altoida NMI is commercially available in all major global healthcare markets and is both, FDA and CE approved. This Phase I/II study is a single-arm, nonrandomized study that is expected to enroll 24 participants. The study's primary objective is to determine the safety and treatment effect of HB-adMSCs for treatment of Alzheimer's disease. HB-adMSCs are administered via four intravenous infusions over an 8-week period and follow-up evaluations every 6 weeks after the last infusion for 1 year. Assessments with Altoida NMI will be performed prior to each infusion and at each follow-up visit thereafter. 


\section{Acquisitions}

Bristol-Myers Squibb \& Celgene

After US regulators finally approved for Bristol-Myers Squibb’s (BMS; NY, USA; www.bms.com) USD 74 billion takeover of Celgene (NJ, USA; www.celgene.com) the acquisition was finally completed on 20 November, more than 10 months after BMS and Celgene first broke the news of the planned merger, and several months after an attempt to scupper the deal by disgruntled investors was defeated [18]. The completed acquisition creates a combined company with annual revenues in excess of USD 34 billion, making it the sector's fourth biggest firm behind Pfizer, Novartis and Roche.

\section{Danaher \& GE Healthcare}

Danaher Corporation (DC, USA; www.danaher.com) is in the process of purchasing the GE Healthcare Life Sciences (IL, USA; www.gelifesciences.com) business units consisting of BioProcess, Cell \& Gene Therapy and Genomics \& Cellular Research [19]. The sale of the GE Healthcare Life Sciences Biopharma business to Danaher is subject to customary closing conditions, including regulatory reviews and appropriate consultation processes, and is expected to close as early as the first quarter of 2020. Once the transaction closes, the company will be named Cytiva.

\section{Capital market \& finances}

\section{Avita}

AVITA Medical (Australia; www.avitamedical.com) has been added to the S\&P/ASX 200 index, effective upon opening of the market on 14 November 2019, 2 months after being added to the S\&P/ASX 300 index [20]. The S\&P/ASX 200 Index is recognized as the institutional investable benchmark in Australia and measures the performance of the largest 200 companies based on float-adjusted market capitalization on the Australian Securities Exchange.

AVITA has also announced an Institutional Placement of USD 83.8 (AUD 120.0) million to fund pipeline development of new indications, including optimizing support for clinical trials and development projects, as well as the Company's continued US commercial growth strategy [21]. The Company has received commitments for AUD 120.0 million at an issue price of USD 0.41 (AUD 0.59) per fully paid ordinary share, representing a $7.2 \%$ discount to the 30 -day volume weighted average price, and a $14.5 \%$ discount to the last closing price on 8 November 2019. The new AVITA shares to be issued under the Institutional Placement will rank equally with existing AVITA shares on issue. The Institutional Placement will occur in a single tranche, and issue 203,389,831 shares to international and Australian investors under its 15\% placement capacity (under ASX Listing Rule 7.1).

\section{Celyad}

Celyad (Belgium; www.celyad.com), a clinical-stage biopharmaceutical company focused on the development of CAR-T cell therapies, has received USD 9.5 (EUR 8.5) million in grants and nondilutive funding from the Walloon Region of Belgium [22]. These funds will help support the development of the Company's CAR-T candidates, including CYAD-01 and CYAD-02 for the treatment of relapsed/refractory acute myeloid leukemia, as well as next-generation approaches currently in preclinical development. Under the terms of this funding from the Walloon Region of Belgium, the Company was awarded a USD 2.7 (EUR 2.4) million grant and nondilutive funding in the form of recoverable cash advances for USD 6.8 (EUR 6.1) million. The regional funding is associated with the Company's specific research and development programs. Under the applicable conditions, the recoverable cash advance is reimbursable over the economic life of the projects. Thirty percent is refundable based on a fixed reimbursement schedule varying between 20 and 25 years, while the balance is refunded under the form of royalties over the same period.

\section{CRISPR Therapeutics}

CRISPR Therapeutics (Switzerland; www.crisprtx.com) has announced the pricing of an underwritten public offering of 4,250,000 common shares at a public offering price of USD 64.50 per share [23]. In addition, the underwriters have a 30-day option to purchase up to an additional 637,500 common shares at the public offering price less the underwriting discount. CRISPR Therapeutics anticipates its gross proceeds from the offering, before deducting underwriting discounts and commissions and other offering expenses, to be approximately USD 
274.1 million, excluding any exercise of the underwriters' option to purchase additional shares. The offering is expected to close on or about 25 November 2019, subject to customary closing conditions.

\section{Financial \& competing interests disclosure}

The authors have received an honorarium from Future Science Group for the contribution of this work. The authors have no other relevant affiliations or financial involvement with any organization or entity with a financial interest in or financial conflict with the subject matter or materials discussed in the manuscript apart from those disclosed.

No writing assistance was utilized in the production of this manuscript.

\section{References}

1. Allogene. Allogene Therapeutics and Notch Therapeutics announce collaboration to research and develop induced pluripotent stem cell (iPSC)-derived allogeneic therapies for hematologic cancer indications (2019). https://ir.allogene.com/node/7101/pdf

2. AVITA Medical. AVITA medical and the gates center for regenerative medicine at the University of Colorado Anschutz Medical Campus enter into collaboration to explore potential spray-on treatment of genetically modified cells for epidermolysis bullosa (2019). www.avitamedical.com/uploads/pdf/AVH-Press-Release-EB-CU-Denver-Partnership-25NOV2019-FINAL.pdf

3. Bluebird Bio. Bluebird Bio and Forty Seven announce a research collaboration to study an all antibody conditioning regimen for use in combination with autologous lentiviral vector hematopoietic stem cell gene therapy (2019). http:

//investor.bluebirdbio.com/news-releases/news-release-details/bluebird-bio-and-forty-seven-announce-research-collaboration

4. Editas Medicine. Editas Medicine and Celgene Corporation amend existing collaboration to develop and commercialize autologous and allogeneic T cell medicines for the treatment of cancer and autoimmune diseases (2019). https://ir.editasmedicine.com/node/9611/pdf

5. NovoHeart. Novoheart to co-develop first of its kind human heart-in-a-jar model of heart failure with AstraZeneca (2019). www.novoheart.com/hk/press/detail/54

6. Tmunity Therapeutics. Tmunity and Oncora Medical partner to advance the use of real-world data to accelerate the availability of CAR-T therapies for cancer patients (2019). www.tmunity.com/2019nov4-tmunity-and-oncora

7. Takeda Pharmaceuticals. Takeda and MD Anderson announce collaboration to accelerate the development of clinical-stage, off-the-shelf car nk-cell therapy platform (2019). www.takeda.com/newsroom/newsreleases/2019/takeda-and-md-anderson-announce-collaborationto-accelerate-the--development-of-clinical-stage-off-the-shelf-car-nk-cell-therapy-platform/

8. Emulate. Liver-Chip predicts human toxicity that was undetected in animal studies for drugs halted in clinical trials (2019). www.emulatebio.com/press/emulate-liver-chip-predicts-human

9. Jang KJ, Otieno MA, Ronxhi J et al. Reproducing human and cross-species drug toxicities using a Liver-Chip. Sci. Transl. Med. 11(517), eaax5516 (2019).

10. Pharmaceutical Technology. First LSCD patient in UK receives Chiesis Holoclar (2019). www.pharmaceutical-technology.com/news/chiesi-holoclar-nhs/

11. HemaCare Corporation. HemaCare expands its product portfolio with GMPrime ${ }^{T M}$ bone marrow aspirate (2019). www.hemacare.com/news/hemacare-expands-its-product-portfolio-with-gmprimetm-bone-marrow-aspirate/

12. Kiadis Pharma. Kiadis Pharma changes strategy to focus solely on development of natural killer (NK) cell therapeutics and terminates development of ATIR101 (2019). www.kiadis.com/for-investors/press-releases/single-press-release/?1945703

13. Takara Bio USA. Takara Bio announces launch of human iPSC-derived intestinal epithelial cells for drug discovery research (2019). www.takarabio.com/about/announcements/takara-bio-announces-launch-of-human-ipsc-derived-intestinal-epithelial-cells-for-drug-d iscovery-research

14. Lineage Cell Therapeutics. Lineage cell therapeutics provides update on SCiStar Clinical Study and OPC1 spinal cord injury program (2019). https://investor.lineagecell.com/node/20186/pdf

15. CRISPR Therapeutics. CRISPR Therapeutics and vertex announce positive safety and efficacy data from first two patients treated with investigational CRISPR/Cas9 Gene-Editing Therapy CTX001 ${ }^{\circledR}$ for severe hemoglobinopathies (2019) http://ir.crisprtx.com/node/9146/pdf

16. Cartesian Therapeutics. Cartesian initiates CAR-T clinical trial in myasthenia gravis. (2019). www.cartesiantherapeutics.com/cartesian-initiates-car-t-clinical-trial-in-myasthenia-gravis/

17. Hope Biosciences. Hope Biosciences receives FDA approval to commence groundbreaking stem cell clinical trial for Alzheimer's disease (2019). www.hope.bio/post/hope-biosciences-receives-fda-approval-to-commence-groundbreaking-clinical-trial-for-alzheimer-s

18. Taylor P. Updated: BMS completes Celgene merger after FTC okay (2019). http://www.pmlive.com/pharma_news/updated_bms_completes_celgene_merger_after_ftc_okay_1317299

19. GE Healthcare. Pre-notification of change in Legal Entity and company name (2019). https://landing1.gehealthcare.com/rs/005-SHS-767/images/Pre-notification $\% 20$ of $\% 20$ change $\% 20$ in $\% 20$ legal\%20entity $\% 20$ and $\% 20$ company\%20name\%20UK\%2020191023\%20Final.pdf 
20. AVITA Medical. AVITA Medical added to S\&P/ASX 200

Index (2019). www.avitamedical.com/uploads/pdf/AVH-Press-Release-ASX-200-07Nov2019-FINAL.pdf

21. AVITA Medical. AVITA Medical announces institutional placement of $A \$ 120.0$ million to support commercial growth and pipeline development (2019). www.avitamedical.com/uploads/pdf/AVH-Press-Release-Equity-Placement-12Nov2019-FINAL-B.pdf

22. Celyad. Celyad receives $€ 8.5$ million in grants and non-dilutive funding by the Walloon Region (2019). www.celyad.com/en/news/celyad-receives-8-5-million-in-grants-and-non-dilutive-funding-by-the-walloon-region

23. CRISPR Therapeutics. CRISPR Therapeutics announces pricing of public offering of common shares (2019). http://ir.crisprtx.com/node/9186/pdf 
